\title{
Energy considerations in a micromagnetic hysteresis model and the Preisach model
}

\author{
Ben Van de Wiele(1),* Lode Vandenbossche ${ }^{(1)}$, Luc Dupré(1), and Daniël De Zutter ${ }^{(2)}$ \\ (1) Department of Electrical Energy, Systems and Automation \\ Ghent University, Sint-Pietersnieuwstraat 41, B-9000 Gent, Belgium. and \\ (2) Department of Information Technology, \\ Ghent University, Sint-Pietersnieuwstraat 41, \\ B-9000 Gent, Belgium.
}

\begin{abstract}
Finding the relations between the microstructural material parameters and the macroscopic hysteresis behavior is indispensable in the design of ferromagnetic materials with minimal (hysteresis) losses. Micromagnetic hysteresis simulations enable a rigorous and structured investigation of these relations since in the numerical model each material parameter can be altered independently. This paper describes a procedure to extract the Preisach distribution function, quantifying the macroscopic hysteresis properties, from micromagnetic simulations incorporating the materials' microstructure. Furthermore, the instantaneously added, stored and dissipated energy while running through the hysteresis loop as described in the macroscopic Preisach model and in the micromagnetic hysteresis model are compared, evidencing a very good agreement. Moreover, using the micromagnetic model, the energy rearrangements between the different micromagnetic interaction terms is studied at each time point of the hysteresis loop. It is concluded that the micromagnetic hysteresis model is a valuable tool in the study of hysteresis properties and loss mechanisms in ferromagnetic materials.
\end{abstract}

\section{INTRODUCTION}

The magnetic hysteresis properties of ferromagnetic materials largely influence their applicability. More precisely, the hysteresis losses determine the efficiency of e.g. electrical machines and transformers $[1,2]$. Therefore, it is indispensable to get a better insight in the loss mechanisms when running through the hysteresis loop and find the microscopic, physical basis of the processes which determine the hysteresis properties. Different approaches have been applied to develop hysteresis models that accurately incorporate the influence of the different microstructural entities of ferromagnetic materials. The models aim at accurately describing the macroscopic magnetization curves using a minimum number of model parameters.

The Jiles-Atherton model [3] uses a differential equation with few model parameters to describes the magnetization curves. In the model developed by Hauser [4] the Gibbs free energy is determined based on statistical grounds and minimized to obtain magnetization curves. Harrison [5] models first-order return curves based on analytical formula describing quantum mechanical processes on the atomistic length scale and classical magnetic processes on the domain length scale. In the Preisach formalism $[6,7]$, different distribution functions defined by different model parameters can be used to map the magnetic properties of the ferromagnetic material. Since in most of these models it is difficult to determine the influence of the various material parameters based on purely theoretical grounds, fitting of the parameters to experimentally results is required. The influence of the

*Electronic address: ben.vandewiele@ugent.be microstructural material properties on the macroscopic magnetic (hysteresis) properties is studied by relating parameters defining the microstructure of the material to model parameters [8-11]. In this approach, a complete, structured study is hardly feasible since it is very difficult to prepare ferromagnetic samples where all microstructural parameters are altered independently to reveal the influence on the model parameters and the hysteresis properties of each distinct parameter.

The micromagnetic formalism [12, 13] describes the magnetization processes based on the (changing) Gibbs free energy in the system, starting from the microstructural properties and the externally applied field. Since the theory is based on the physical interactions on the nanometer space scale, the only parameters are known material constants which excludes the need experiments and fitting. Moreover, the theory is applicable for all magnetic materials where most models are only applicable for a certain class of magnetic materials. This makes a micromagnetic hysteresis model as [14] ideally suited to study the relations between microstructure and magnetic hysteresis properties.

In many applications, one aims at designing ferromagnetic materials with minimal hysteresis losses. In this framework one should be able to determine which material parameters influence the losses, not only integrated, after running through the complete hysteresis loop, but also instantaneously, on each given time point of the loop. Moreover, one should be able to distinguish the instantaneous energy arrangements in the ferromagnetic material. Macroscopically, it is only possible to determine (i) the total dissipated energy during one cycle as the surface of the hysteresis loop and (ii) instantaneous energy which is added to the ferromagnetic material [15]. Since the micromagnetic hysteresis model is based on the (changing) Gibbs free energy in the system, it is able to 
determine also the instantaneously added and stored energy as well as the mutual instantaneous energy flows between the micromagnetic energy terms. Enabling the determination of the energy flows from, to and in the micromagnetic sample, a micromagnetic hysteresis model is ideally suited to gather a greater insight in the hysteresis mechanisms in ferromagnetic materials.

Also in the Preisach formalism, the instantaneously added, stored and dissipated energy can be determined starting from a given Preisach distribution function (PDF). In this paper, we present a strategy to obtain the PDF from micromagnetic simulations. Based on this PDF we compare and interpret the instantaneous energy flows as described in the Preisach and the micromagnetic approach. Furthermore, we study the instantaneous energy rearrangements in the material using the micromagnetic hysteresis model.

\section{MICROMAGNETIC HYSTERESIS MODEL}

The used micromagnetic hysteresis model is extensively described in [14]. Here we will only provide a short summary. The ferromagnetic object is discretized using cubical finite difference cells of identical shape. According to the micromagnetic theory [13], the magnetization in each cell is considered to be uniform with a fixed amplitude $M_{s}$ and a varying orientation. This defines the magnetization field $\mathbf{M}(\mathbf{r}, t)=M_{s} \mathbf{m}(\mathbf{r}, t)$. In the hysteresis model time is discretized on two different time scales, see Fig. 1. The externally applied field $H_{a}$ is approximated by a staircase function varying on a macroscopic time scale $\Delta t$. It is guaranteed that at the moment the applied field jumps from one constant value $H_{a}$ to the next one $H_{a}+\Delta H_{a}$, the ferromagnetic system is in static micromagnetic equilibrium: each equilibrium magnetization state is determined by time stepping the Landau-Lifshitz (LL) equation, describing the fast dynamics of the magnetization field $\mathbf{m}(\mathbf{r}, t)$ on a picosecond time scale, in each FD cell until the next micromagnetic equilibrium state is reached. For time stepping the LLequation a microscopic time step $\delta t \ll \Delta t$ is used. To check wether the next equilibrium state is reached, the following stopping criterium is verified for all FD cells

$$
\frac{\left|\mathbf{m}\left(t_{i}\right)-\mathbf{m}\left(t_{i}+\delta t\right)\right|}{\delta t}<\epsilon .
$$

With $\delta t$ expressed in ps, a typical value of $\epsilon$ is 0.01 . Other groups [16] choose to apply a zero torque stopping criterium which is equivalent due to the structure of LLequation. However, the use of criterium (1) enables to easier distinguish equilibrium states when thermal effects are incorporated through a randomly fluctuating thermal field $\mathbf{H}_{t h}$, added to the effective field [17]. Indeed, at any time point, $\mathbf{H}_{t h}$ gives rise to a fluctuating local torque $\propto \mathbf{m} \times \mathbf{H}_{t h}$ which can be larger than a predefined threshold while the resulting change in local magnetization $\left|\mathbf{m}\left(t_{i}\right)-\mathbf{m}\left(t_{i}+\delta t\right)\right| / \delta t$ can be negligible, indicating

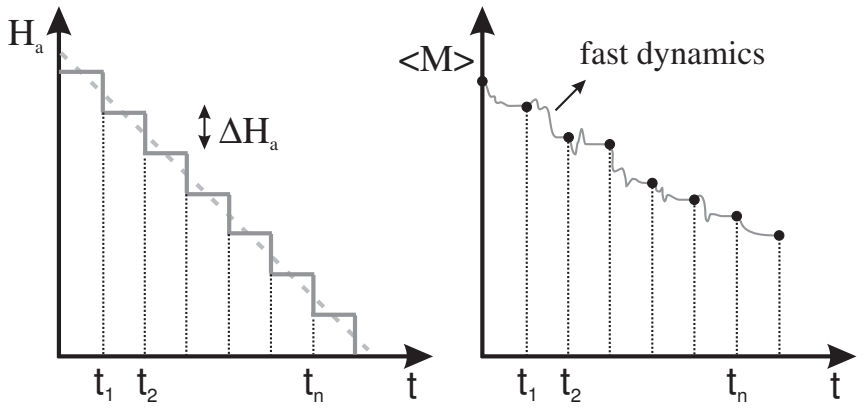

FIG. 1: The time evolution of the applied field is discretized using macroscopic time steps $\Delta t$ between time points $t_{i+1}=$ $t_{i}+\Delta t$ at which the system is in an equilibrium state. The fast magnetization dynamics describing the evolution between equilibrium points is discretized using order picosecond time steps $\delta t$.

that an equilibrium state is reached.

Once the criterium (1) is met in all FD cells simultaneously, the equilibrium state is reached and the applied field takes a next jump for which a successive magnetization state is computed, etc. The macroscopic transition curves are obtained from the micromagnetic computations by considering the mean magnetization $<\mathbf{M}>=M_{s}<\mathbf{m}>$ at the successive equilibrium points at time points $t_{1}, t_{2}, \ldots$, see Fig. 1 .

Note that time stepping the LL-equation guarantees that, out of the many equilibrium states possibly corresponding with a particular applied field, only those successive equilibrium states are selected which can be reached starting from the last considered equilibrium state, i.e. with ever decreasing Gibbs free energy path between two successive states. Hence, the micromagnetic hysteresis approach inherently incorporates the magnetization history. This is in contrary to hysteresis schemes based on the direct minimization of the Gibbs free energy where one needs to evaluate the direction of the energy gradient to determine the path along which to move to the next energy equilibrium. In such an approach, making a reliable estimate of the gradient in the rich structure of the micromagnetic energy landscape is far from obvious [15].

We also remind that, when considering micromagnetic hysteresis computations on large ferromagnetic objects, special attention should go to the effectiveness of the used time stepping scheme. The scheme should incorporate the three main properties of the LL-equation: (i) preservation of the magnetization field amplitude, (ii) the decrease in total Gibbs free energy under a constant applied field and (iii) conservation of the Gibbs free energy in case of zero damping. Classical 'off-the-shelf' methods as the Euler method, Heun method or Runge Kutta methods do not preserve the magnetization amplitude and violate the other properties leading to possibly incorrect results. Many geometrical integrators [18-21] are developed to preserve the magnetization amplitude by 
exploiting symmetries, invariant quantities, hamiltonian structures etc. of the LL-equation. However, not all of them meet the two other properties. A geometrical integration method based on the mid point rule described in [22] incorporates all three properties and is unconditionally stable. However, being an implicit method, a large system of coupled non-linear equations needs to be solved every time step which limits its application for large ferromagnetic systems. Therefore we opt to use the semi-analytical predictor-corrector scheme [23]. Being an explicit time stepping scheme, it does not require solving a set of coupled equations. The method has a high order convergence rate and meets the three intrinsic properties of the LL-equation, enabling the use of large time steps $\delta t$.

Furthermore, when considering large material samples, special attention should go to the fast evaluation of the magnetostatic field. Fast multipole method (FMM) based algorithms as [24] are very flexible regarding the used discretization and can incorporate an adaptive meshing [25]. Fast Fourier transform (FFT) based algorithms are restricted to a regular discretization grid. Given such a regular grid, they are much faster then FMM based schemes, but require more memory resources [26]. The fast Fourier transform on multipoles (FFTM) technique [27] developed by Ong et al. is a combination of the FMM and FFT method. It still needs a regular grid, but shows more flexibility -adaptive discretizationcompared to a pure FFT scheme. For the regular discretization grid with identical FD cells applied here, the FFT based scheme is used since it outperforms all other evaluation techniques when CPU time is considered.

\section{CLASSICAL PREISACH HYSTERESIS MODEL}

We again restrict ourselves to a short summary of the model and refer to reference text books for a complete description of the model $[7,15]$. In the classical Preisach model [6], the description of uniaxial rate independent hysteresis properties is based on an infinite collection of elementary magnetic dipoles . The dipoles have an asymmetric rectangular hysteresis loop with switching fields commonly denoted by $\alpha$ and $\beta(\alpha \geq \beta)$. As shown in Fig. 2, the polarity of a given dipole $(\alpha, \beta)$ depends on the value of the applied field $H_{a}$ and/or its history $H_{a, h i s t}$. For $H_{a}>\alpha$ the polarity $\eta$ is +1 . When $H_{a}$ is now decreased, the polarity changes to -1 at $H_{a}=\beta$. The increasing branch of the dipole defines the polarity for increasing $H_{a}$ values. Here, the polarity switches from -1 to +1 at $H_{a}=\alpha$.

To describe the hysteretic magnetic behavior of a ferromagnetic sample, first the reversible and the irreversible part of the magnetization loop is separated. The irreversible part is then represented by the Preisach distribution function (PDF) $P(\alpha, \beta)$, which corresponds to the statistical density of elementary dipoles $(\alpha, \beta)$. The mag-

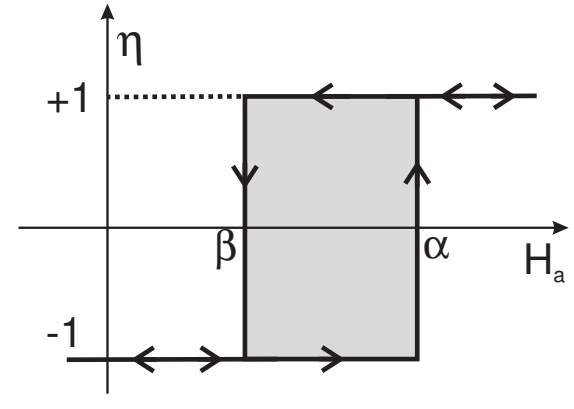

FIG. 2: Elementary Preisach dipole with switching fields $\alpha$ and $\beta$.

netization in the Preisach model is now expressed as an integral over the entire elementary dipole collection

$$
\begin{aligned}
M\left(H_{a}\right)= & M_{\text {rev }}\left(H_{a}\right)+M_{i r r}\left(H_{a}, H_{a, h i s t}\right) \\
M_{i r r}\left(H_{a}\right)= & \frac{1}{2 \mu_{0}} \int_{-\infty}^{+\infty} \mathrm{d} \alpha \\
& \int_{-\infty}^{\alpha} \mathrm{d} \beta \eta\left(\alpha, \beta, H_{a}, H_{a, \text { hist }}\right) P(\alpha, \beta) .
\end{aligned}
$$

The $\operatorname{PDF} P(\alpha, \beta)$ is shown to be dependent on the microstructure of the ferromagnetic sample [11, 28, 29] and can be interpreted as a blueprint of the magnetic hysteretic properties of a given sample.

\section{OBTAINING THE PDF FROM MICROMAGNETIC SIMULATIONS}

The PDF is typically extracted from a whole set of measured quasi-static first-order symmetric magnetization loops, from low to high magnetic induction levels, starting on the virgin curve or from a number of first order reversal curves (FORCs) covering the complete range of the major hysteresis loop [7]. In the micromagnetic simulations, magnetization branches should always start at a known equilibrium state. The demagnetized state at which the virgin curve emanates is not known, nor easy to obtain, while FORCs emanate at known micromagnetic equilibrium states on the major hysteresis loop. Therefore, the PDF is extracted from FORCs.

In the micromagnetic simulations, by way of example, a sample is considered with dimensions $0.32 \mu \mathrm{m} \times 0.32 \mu \mathrm{m} \times 5.12 \mu \mathrm{m}$. The sample comprises 16 grains in the elongated direction with random cubical easy axes orientations. The saturation polarization is $\mu_{0} M_{s}=2.16 \mathrm{~T}$, the exchange stiffness is small: $0.2510^{-12} \mathrm{~J} / \mathrm{m}$ and the anisotropy constants are $K_{1}=$ $0.4810^{6} \mathrm{~J} / \mathrm{m}^{3}$ and $K_{2}=-0.5010^{5} \mathrm{~J} / \mathrm{m}^{3}$. The external field is applied in the elongated direction of the sample and ranges from $3.010^{6} \mathrm{~A} / \mathrm{m}$ to $-3.010^{6} \mathrm{~A} / \mathrm{m}$ with steps of $\Delta H_{a}=400 \mathrm{~A} / \mathrm{m}$. The simulated FORCs are shown in Fig. 3. For this simple system, the small exchange 


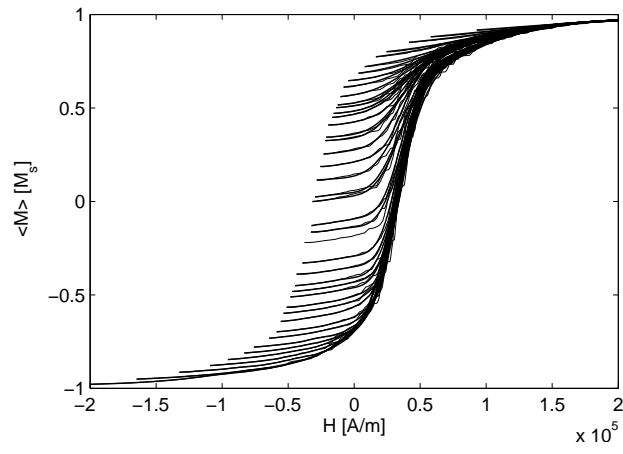

FIG. 3: Simulated FORCs using the micromagnetic hysteresis scheme.

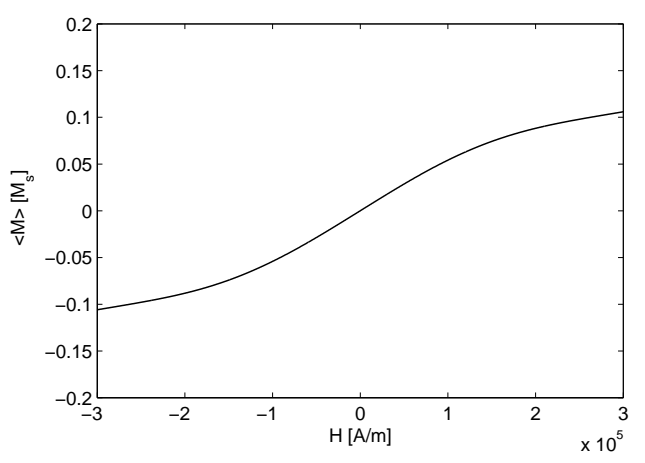

FIG. 4: Reversible part of the magnetization curves corresponding to Fig. 3.

stiffness ensures that the hysteresis loop is smooth and does not show too large magnetization jumps for varying $H_{a}$. In this way, FORCs can start at relatively equidistant steps in the magnetization. In larger, more complex, non-ideal materials, the smoothness of the loop is guaranteed by the presence of various pinning sites as impurities in the iron lattice.

While this simulation is restricted to a very simplified sample, the following analysis is representative since the approach is identical for more complex materials. For those non-ideal materials, the local material parameters have to be determined on the used discretization scale. The used micromagnetic hysteresis model is directly applicable to the obtained more real world, complex input. Moreover, in the micromagnetic hysteresis model, the influence of impurities like dislocations, voids, interstitials, etc. is included by taking into account the magneto-elastic interaction between their local characteristic stress distributions and the local magnetization [30]. Given the input parameters, the evaluation of the effective field contributions stays unaltered and, consequently, the treatment of the different energy contributions. Since no impurities are treated in the considered sample, the magneto-elastic field is zero.
The determination of the PDF now encompasses four steps.

1. The numerical magnetization curves are smoothed using splines which meet the criterion $\mathrm{d} M / \mathrm{d} H_{a}>0$, required in the next steps.

2. Following equations (2) and (3), the reversible magnetization processes $M_{\text {rev }}\left(H_{a}\right)$ are extracted by considering the differential permeability at the reversal points. At these points only reversible magnetization processes occur and thus the reversible differential permeability $\mu_{\text {rev }}\left(H_{a}\right)$ can be defined. From this, the single valued magnetization curve $M_{\text {rev }}\left(H_{a}\right)$ is deduced

$$
M_{\text {rev }}\left(H_{a}\right)=\int_{0}^{H_{a}}\left(\frac{\mu_{r e v}(H)}{\mu_{0}}-1\right) \mathrm{d} H .
$$

Consequently the irreversible magnetization curves are

$$
M_{i r r}\left(H_{a}\right)=M\left(H_{a}\right)-M_{r e v}\left(H_{a}\right) .
$$

The reversible part of the magnetization curves is shown in Fig. 4.

3. The set of irreversible magnetization curves $M_{i r r}\left(H_{a}\right)$ is now rearranged into an Everett map [31] The Everett function $E_{e v}(\alpha, \beta)$ is defined as

$$
E_{e v}(\alpha, \beta)=\mu_{0}\left|M_{i r r}(\beta)-M_{i r r}(\alpha)\right|
$$

with $\alpha$ an extremal value for the applied field $H_{a}$, and no extrema are evaded from the memory when passing from $\alpha$ to $\beta$.

4. Ultimately, the PDF is obtained by differentiating the Everett function

$$
P(\alpha, \beta)=-\frac{\partial^{2} E_{e v}(\alpha, \beta)}{\partial \alpha \partial \beta} .
$$

The PDF is shown in Fig. 5. Note that the PDF has only non-zero values in the half plane $\alpha>\beta$. Furthermore, the constructed PDF has small areas with nonphysical negative values. These originate in the crossing of the micromagnetically simulated FORCs in the region close to the coercive field of the limit cycle (see Fig. 3).

To test the accuracy of the extracted PDF, the major hysteresis loop is reconstructed from the PDF and compared to the original hysteresis loop obtained from the micromagnetic simulations. Figure 6 shows the excellent agreement between the loop reconstructed from the PDF data and the original micromagnetically simulated hysteresis loop: the reconstructed loop is a smoothed version of the micromagnetic hysteresis loop which shows Barkhausen jumps. Indeed, the micromagnetic model is able to describe the discontinuous movement of the domain walls resulting in the Barkhausen jumps while the Preisach model describes macroscopically averaged -and thus more continuous- magnetization processes.

Note that this procedure can be applied rigorously to find relations between the microstructural material parameters and the PDF in a structured way. When e.g. a Lorentzian distribution function is fitted to the PDF, relations between the parameters describing the Lorentzian distribution and the microstructural parameters can be investigated. 


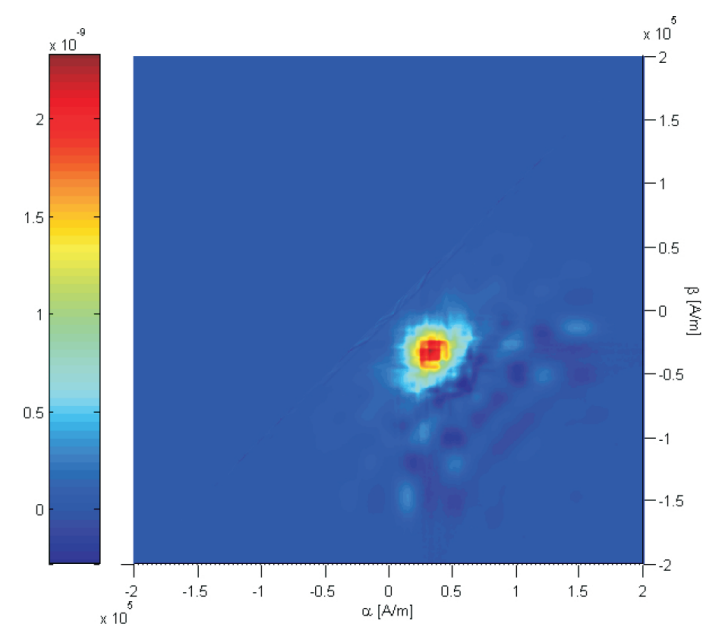

FIG. 5: Reconstructed Preisach distribution function, based on the smoothed irreversible first order reversal curves of Fig. 4 .

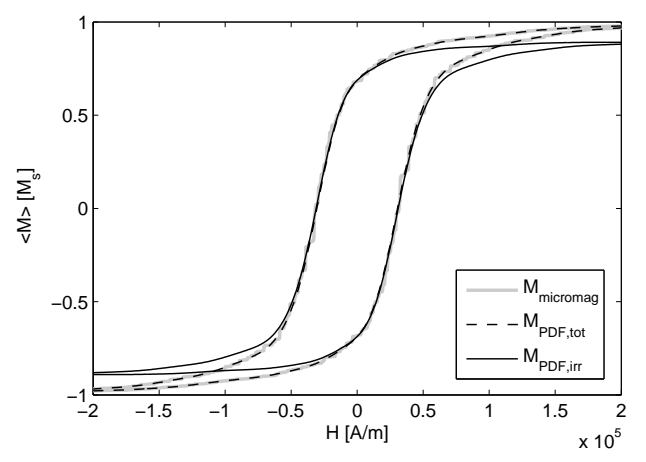

FIG. 6: Simulated hysteresis loop compared with the hysteresis loop reconstructed from the PDF. For completeness, the irreversible hysteresis loop is also shown.

\section{ENERGY DESCRIPTION}

\section{A. Energy flows in the micromagnetic hysteresis model}

The energy variations as described by the micromagnetic hysteresis model during the transition between two successive equilibrium states is sketched in Fig. 7. At a given time point $t_{i}$, an external field $H_{a}$ is applied and a corresponding equilibrium magnetization configuration $\mathbf{m}\left(t_{i}\right)$ is reached, state A. Now, the applied field takes a jump $\Delta H_{a}$. This increases the energy in the system to a state $\mathrm{B}$, which is unstable. Consequently, the original magnetization configuration $\mathbf{m}\left(t_{i}\right)$ evolves towards a new equilibrium magnetization configuration $\mathbf{m}\left(t_{i}+\Delta t\right)$ corresponding to state $\mathrm{C}$. This new equilibrium state $\mathrm{C}$ has a lower energy compared to the unstable state B. During the transition from state $\mathrm{B}$ to state $\mathrm{C}$, the difference in Gibbs free energy $\Delta \phi_{G}$ is dissipated as heat towards

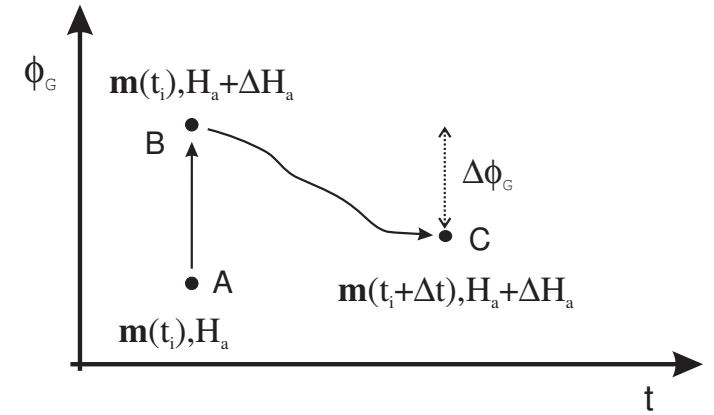

FIG. 7: Dissipated energy density in the micromagnetic hysteresis model: the difference in Gibbs free energy $\Delta \phi_{G}$ corresponds to the dissipated energy density.

the thermal bath. Hence, the dissipated energy density $\Delta \phi_{\text {diss }}$ equals $\Delta \phi_{G}$.

The added energy density $\Delta \phi_{a d d}$ can be derived from the macroscopic formula as

$$
\Delta \phi_{a d d}=\mathbf{H}_{a} \Delta \mathbf{B}=\mu_{0} \mathbf{H}_{a} \cdot\left(\Delta \mathbf{M}+\Delta \mathbf{H}_{a}\right),
$$

In the used model, the applied field is constant between two equilibrium states and equals $\mathbf{H}_{a}+\Delta \mathbf{H}_{a}$. Hence in terms of the micromagnetic quantities used in Fig. 7 the added energy density yields

$$
\begin{aligned}
\Delta \phi_{a d d}= & \mu_{0} M_{s}\left(\mathbf{H}_{a}+\Delta \mathbf{H}_{a}\right) \cdot\left[<\mathbf{m}\left(t_{i}+\Delta t\right)>-<\mathbf{m}\left(t_{i}\right)>\right] \\
& +\mu_{0}\left(H_{a}+\Delta H_{a}\right) \Delta H_{a} .
\end{aligned}
$$

The conservation of energy principle then yields the stored energy density as

$$
\Delta \phi_{s t}=\Delta \phi_{a d d}-\Delta \phi_{d i s s}
$$

Note that in the micromagnetic hysteresis model each micromagnetic energy term can be studied in detail. Indeed, inspection of the differences in the Zeeman, exchange, anisotropy and magnetostatic energy between state $\mathrm{B}$ and state $\mathrm{C}$ yield the energy rearrangements between the different micromagnetic energy terms.

\section{B. Energy flows in the classical Preisach model}

In the classical Preisach model, one can distinguish the instantaneous energy density that is added, stored and dissipated when running through the hysteresis loop by inspecting the elementary Preisach dipole. The following energy densities can be associated with the areas shown in Fig. 8. During the switching process of the ascending branch, the area $(a)+(b)+(c)$ corresponds to the energy density added by the source. From that, the area (c) and the area $(a)+(b)$ correspond to the dissipated and the stored energy density in the system respectively. During the switching process of the descending branch, the area 


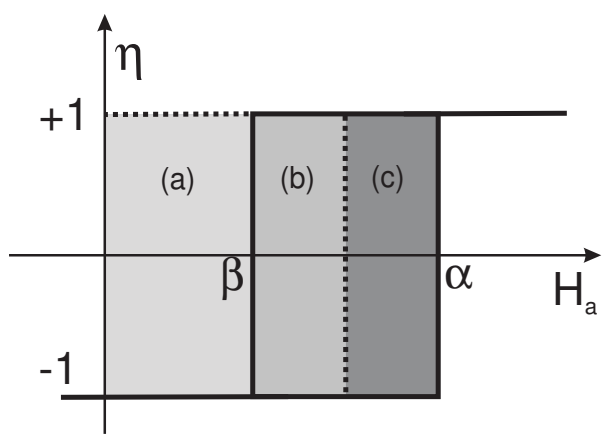

FIG. 8: Elementary Preisach dipole with associated energy densities.

(a) + (b) corresponds to the stored energy density, while the areas (a) and (b) correspond to the energy density which is returned to the source and dissipated by the system respectively.

By extending the above considerations to all dipoles in the PDF that are switching when the applied field is altered, the irreversibly added energy density $\phi_{a d d, i r r}$, the irreversibly stored energy density $\phi_{s t, i r r}$ and the dissipated energy density $\phi_{\text {diss }}$ can be distinguished at each time point of the hysteresis loop. For the ascending branch, an increase $\Delta H_{a}$ of the applied field leads to

$$
\begin{aligned}
& \Delta \phi_{a d d, i r r}=\int_{H_{a}}^{H_{a}+\Delta H_{a}} \mathrm{~d} \alpha \int_{H_{m i n}}^{\alpha} \alpha P(\alpha, \beta) \mathrm{d} \beta \\
& \Delta \phi_{s t, i r r}=\int_{H_{a}}^{H_{a}+\Delta H_{a}} \mathrm{~d} \alpha \int_{H_{m i n}}^{\alpha} \frac{\alpha+\beta}{2} P(\alpha, \beta) \mathrm{d} \beta \\
& \Delta \phi_{\text {diss }}=\int_{H_{a}}^{H_{a}+\Delta H_{a}} \mathrm{~d} \alpha \int_{H_{m i n}}^{\alpha} \frac{\alpha-\beta}{2} P(\alpha, \beta) \mathrm{d} \beta
\end{aligned}
$$

with $H_{\text {min }}$ the last minimum of $H_{a}$ kept in memory. For the descending branch, a decrease $\Delta H_{a}$ of the applied field leads to

$$
\begin{aligned}
& \Delta \phi_{a d d, i r r}=\int_{H_{a}}^{H_{a}-\Delta H_{a}} \mathrm{~d} \beta \int_{\beta}^{H_{\max }} \beta P(\alpha, \beta) \mathrm{d} \alpha \\
& \Delta \phi_{s t, i r r}=\int_{H_{a}}^{H_{a}-\Delta H_{a}} \mathrm{~d} \beta \int_{\beta}^{H_{\max }} \frac{\alpha+\beta}{2} P(\alpha, \beta) \mathrm{d} \alpha \\
& \Delta \phi_{\text {diss }}=\int_{H_{a}}^{H_{a}-\Delta H_{a}} \mathrm{~d} \beta \int_{\beta}^{H_{\max }} \frac{\beta-\alpha}{2} P(\alpha, \beta) \mathrm{d} \alpha,
\end{aligned}
$$

with $H_{\max }$ the last maximum of $H_{a}$ kept in memory. Note that negative values for the added energy density correspond to an energy flow from the system towards the source. Negative values for the stored energy density indicate that more energy is leaving the system (towards the source or by dissipation) than that energy is added to the system. By definition, the dissipated energy is always positive. Further, note that the reversible magnetization processes only have a contribution to the total added and stored energy density

$$
\begin{aligned}
\Delta \phi_{\text {add }, \text { rev }}=\Delta \phi_{\text {st }, \text { rev }} & =\int_{H_{a}}^{H_{a} \pm \Delta H_{a}} H \mathrm{~d} B_{\text {rev }} \\
& =\int_{H_{a}}^{H_{a} \pm \Delta H_{a}} \mu_{r e v}(H) H \mathrm{~d} H .
\end{aligned}
$$

Here, the sign in the integral boundaries is determined by which branch is considered: negative for the descending branch and positive for the ascending branch.

\section{DISCUSSION}

In what follows we compare and comment on the energy flows in the ferromagnetic system when running through the descending branch. Similar conclusions can be drawn when running through the ascending branch.

\section{A. Instantaneous energy flows}

The instantaneously added, dissipated and stored energy densities as determined in the micromagnetic and the Preisach model are shown in Figs. 9, 10 and 11 respectively. The energy density curves derived from the micromagnetic model show a highly irregular behavior. Each spike corresponds to a Barkhausen jump at which large energy flows occur in the ferromagnetic system. Since the Preisach model incorporates a high level of smoothing, the resulting energy density curves are continuous. To facilitate the comparison between the micromagnetic and the Preisach data, a locally averaged version of the micromagnetic energy density curves is added to the figures. In all figures, the coercive field value is indicated with a vertical dash-dotted line. Note that since the descending branch is considered, all curves in the following figures should be interpreted from right to left for increasing time (i.e. from positive to negative applied fields).

\section{Instantaneously added energy}

The instantaneously added energy density in the two models is shown in Fig. 9. While returning from positive saturation to $H_{a}=0.0 \mathrm{~A} / \mathrm{m}$ the added energy is negative, which indicates that energy is flowing from the material towards the source. For negative applied fields, the added energy density is positive, indicating that energy is flowing from the source to the material. The largest amounts of energy are added at applied field values in the proximity of the coercive field. The expressions (14) and (9) used in the Preisach and micromagnetic description respectively result in coinciding curves. 


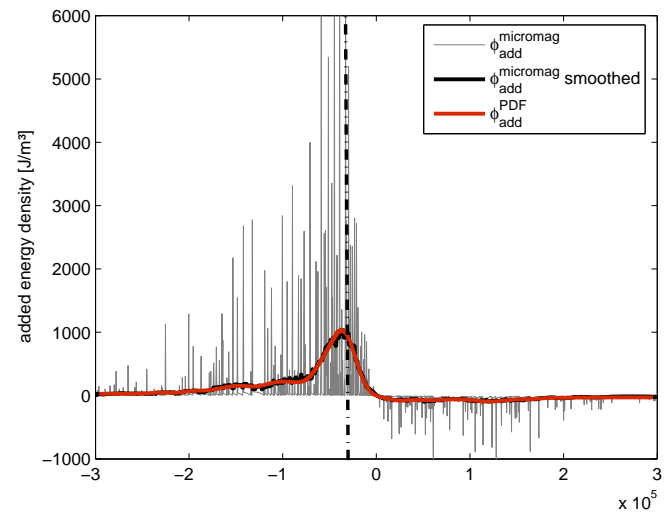

FIG. 9: Instantaneously added energy density when running through the descending branch of the hysteresis loop shown in Fig. The dash-dotted line indicates the coercive field.6.

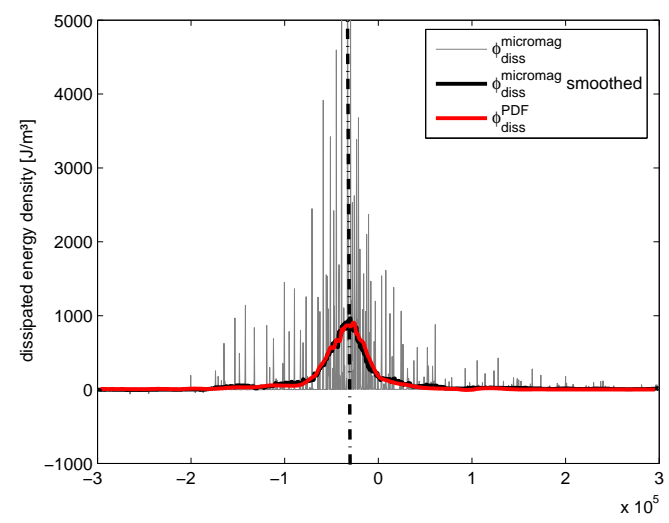

FIG. 10: Instantaneously dissipated energy density when running through the descending branch of the hysteresis loop shown in Fig. The dash-dotted line indicates the coercive field. 6 .

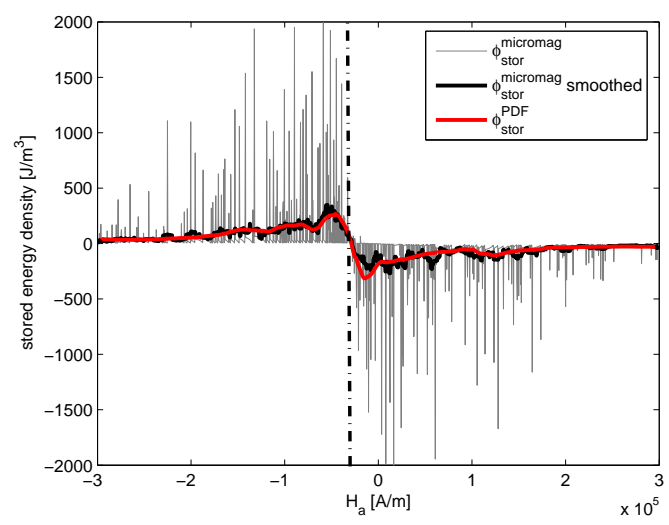

FIG. 11: Instantaneously stored energy density when running through the descending branch of the hysteresis loop shown in Fig. The dash-dotted line indicates the coercive field. 6 .

\section{Instantaneously dissipated energy}

The instantaneously dissipated energy density in the two models is shown in Fig. 10. This energy flow corresponds to energy that is dissipated as heat during the irreversible magnetic processes which occur in the material, i.e. during the Barkhausen jumps. It represents the energy flow from the material towards the thermal bath and is thus per definition always positive. The curves in Fig. 10 have indeed only positive values. In the micromagnetic context, this is a consequence of the LLG equation which guarantees the continuous decrease in energy for constant applied fields. In the Preisach model, the positive values result from the prerequisite $\alpha \geq \beta$ and $P(\alpha, \beta) \geq 0$.

Most of the energy is dissipated for applied field values between $0.0 \mathrm{~A} / \mathrm{m}$ and $-1.010^{5} \mathrm{~A} / \mathrm{m}$ which roughly corresponds to an average magnetization between $\pm 0.8 M_{s}$, see Fig. 6. This shows that most of the magnetic processes in this part of the hysteresis loop are irreversible in nature, while closer to saturation reversible processes are dominant. This is in correspondence with literature [15]. Figure 12 shows the number of time steps required to reach equilibrium at successive constant applied field values in the micromagnetic model. Here a convergence threshold of $\epsilon=0.01$ in the stopping criterium (1). The choice of an other value will lead to proportional peaks. The number of time steps needed to reach equilibrium is related with the 'irreversibility' of the magnetic process. Indeed, since large variations in the magnetization structure can occur during Barkhausen jumps, the simulation of these irreversible magnetization processes require a large number of time steps to come to the new equilibrium points. Only small changes in the magnetization structure occur during reversible magnetization processes, which leads to a small number of time steps required to reach new equilibrium points. In the Preisach description, all energy is dissipated when running through the irreversible hysteresis loop shown in Fig. 6. It is clear that the descending branch of the irreversible hysteresis loop has the largest slope in the range $-1.010^{5} \mathrm{~A} / \mathrm{m}<H_{a}<0.0 \mathrm{~A} / \mathrm{m}$.

\section{Instantaneously stored energy}

The instantaneously stored energy density in the two models is shown in Fig. 11. The agreement between both descriptions is very good. When saturated in the positive direction, a large amount of energy is stored in the system. When reducing the external field, this energy is released towards the source or dissipated as heat giving rise to negative values for the instantaneously stored energy density. When the ferromagnetic system reaches the state corresponding to $\mathbf{M}=0$ and $H_{a}=H_{c}$-further called the coercive state - the system starts to absorb energy when the field is further reduced towards negative saturation. This leads to positive values for the instan- 


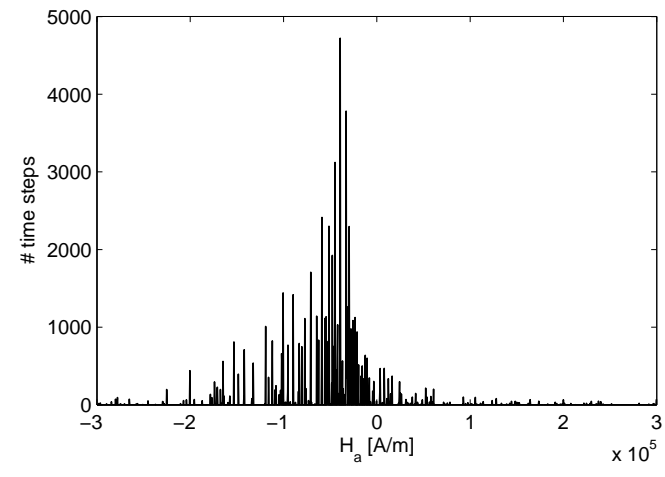

FIG. 12: Number of time steps required to reach equilibrium at successive constant applied field values $H_{a}$ when running through the descending branch from $H_{a}=310^{5} \mathrm{~A} / \mathrm{m}$ to $H_{a}=$ $-310^{5} \mathrm{~A} / \mathrm{m}$.

taneously stored energy density. From these curves, it is clear that at the coercive state no energy is stored in the ferromagnetic system. Starting from this point, any field variation will result in an increase of the stored energy.

\section{B. Instantaneous micromagnetic energy rearrangement}

When studying the loss mechanisms in ferromagnetic materials and how microstructural material parameters influence them, it is instructive to take a closer look at the different micromagnetic energy contributions. In this way it is possible to determine which features (magnetocrystalline effects through the anisotropy term, demagnetizing effects through the magnetostatic field, etc.) are responsible for the largest amount of stored, dissipated and added energy. By changing the microstructural input parameters of the micromagnetic hysteresis simulations, the different energy flows will change when running through the hysteresis loops. Such a study can contribute to the development of improved materials. This investigation is only possible in the micromagnetic model.

In the micromagnetic description of the instantaneously dissipated energy discussed above, the difference in the total Gibbs free energy is considered as sketched in Fig. 7. In the same way one can consider the difference in the Zeeman energy $\phi_{a}$, the magnetostatic energy $\phi_{m s}$, the anisotropy energy $\phi_{a n i}$ and the exchange energy $\phi_{\text {exch }}$ separately to come to the instantaneous flows of these energy contributions. The corresponding instantaneous changes in micromagnetic energy densities $\delta \phi_{a}, \delta \phi_{m s}$, $\delta \phi_{a n i}$ and $\delta \phi_{\text {exch }}$ are shown in Fig. 13. Here, positive values correspond to an instantaneous energy flow from the considered energy term to the other terms and/or the thermal bath while negative values correspond to an instantaneous energy flow towards the considered energy term and thus an increase of the considered energy.
It is clear that, although the total instantaneously dissipated energy is strictly positive, the instantaneous changes in the separate energy densities can have negative values. This indicates that energy is rearranged in the system while running through the hysteresis loop. In the saturated state, the Zeeman and the exchange energy are minimal because the sample is uniformly magnetized along the applied field, while the magnetostatic and the anisotropy energy are large since the flux lines do not close in the ferromagnetic material and the local magnetization in the material is not aligned along an easy axis. At the coercive field however, the magnetostatic and anisotropy energy are very small and the Zeeman and exchange energy are large since the system now has a magnetization state with closed flux lines and with the local magnetization mainly aligned along a local anisotropy axis. Hence, depending on the applied field, energy is rearranged in the ferromagnetic material. Based on the plots in Fig. 9 we will now comment on these energy rearrangements when running through the hysteresis loop:

- The Zeeman energy has the largest contribution to the total dissipated energy. The largest flows of Zeeman energy occur around the coercive field where the slope of the hysteresis loop is maximal. In this part of the hysteresis loop large portions of the ferromagnetic material are aligning with the applied field and the Zeeman energy is vastly reduced. Here, Zeeman energy is dissipated as heat or stored as magnetostatic, anisotropy and/or exchange energy.

- The magnetostatic energy flow is positive until the coercive magnetization state is reached. Hence, while running through this part of the hysteresis loop magnetostatic energy is converted to heat and/or other micromagnetic energy contributions. The coercive state contains the minimal magnetostatic energy since all flux lines close in the material and only small demagnetizing effects appear in the domain walls. When going further to saturation, the net magnetization along the applied field direction grows together with the demagnetizing fields, leading to an increase of the magnetostatic energy and thus negative values for the instantaneous change in magnetostatic energy density $\delta \phi_{m s}$.

- The instantaneous anisotropy energy flow has large contributions over a relatively broad range of the hysteresis loop. Whenever a region in the magnetic sample is aligning with a local easy axis, the anisotropy energy decreases leading to positive values for the anisotropy energy flow (when going to the coercive state). Whenever the magnetization in a region is rotating away from a local easy axis, anisotropy energy is built up (when going to saturation). 

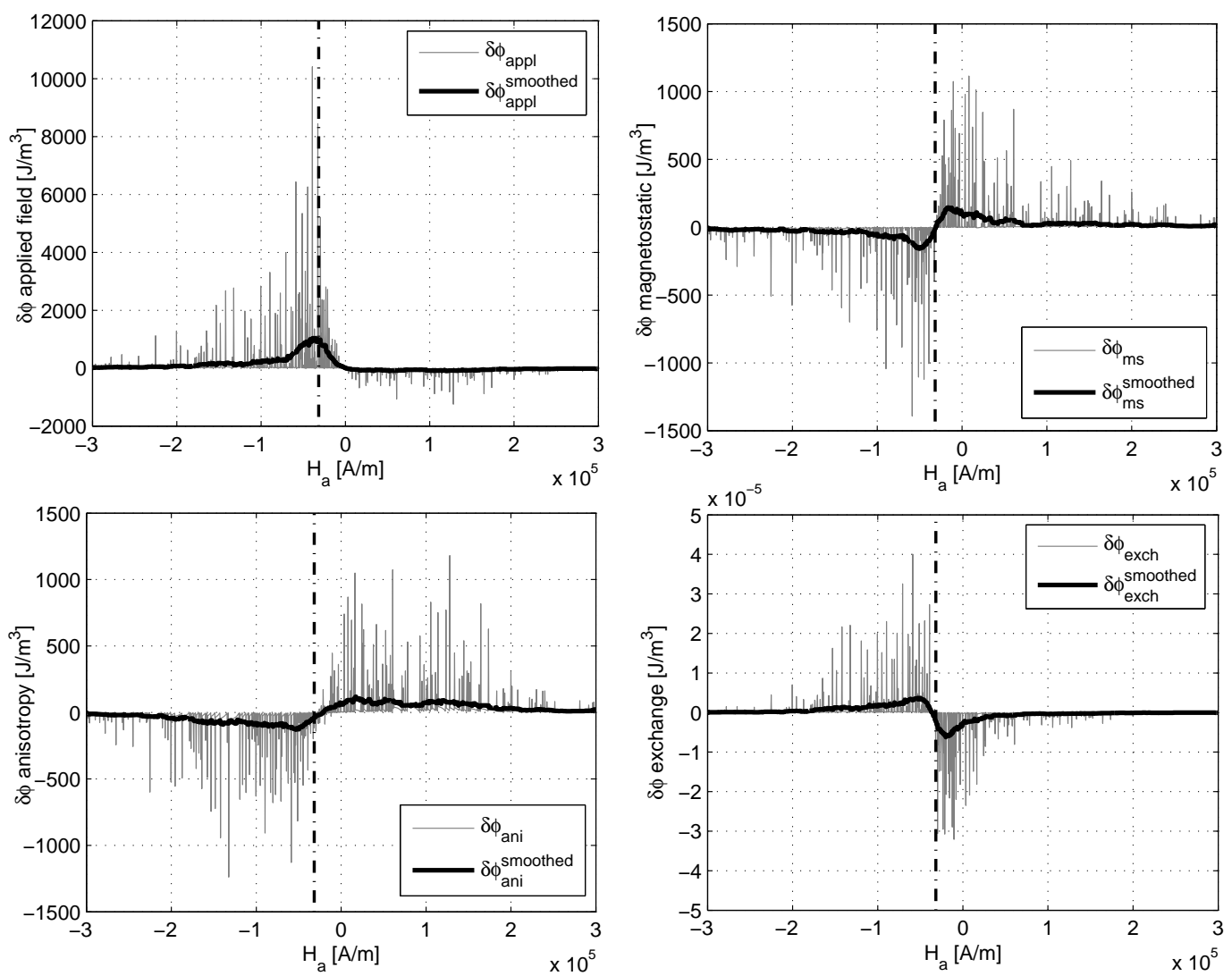

FIG. 13: Instantaneous changes in energy density for the different micromagnetic energy terms. From left-top to right-bottom: Zeeman energy density, magnetostatic energy density, anisotropy energy density and exchange energy density.

- In the considered system, the exchange energy is negligible since the exchange stiffness $A$ is very small. This was chosen so in order to have a nonstiff system leading to a smooth hysteresis loop with only small magnetization jumps, which was a prerequisite to have FORCs emanating at relatively equidistant magnetization values $\langle M\rangle$, see Fig. 3. Although the exchange energy flows are negligible, the curve $\delta \phi_{\text {exch }}$ is still instructive. It is clear that the amplitude of the dissipated exchange energy is proportional to the slope of the hysteresis loop. The exchange energy is built up until the coercive magnetization state is reached. This coercive state has the most non-uniform equilibrium domain configuration when running through the hysteresis loop. Indeed, at this point the exchange energy density is maximal. When going further to saturation the sample gradually becomes more uniformly magnetized, leading to energy flows towards the thermal bath and the other energy terms.

\section{Cumulated energy flows}

The cumulated added, dissipated and stored energy densities based on the Preisach description are shown in Fig. 14. The curves obtained from the micromagnetic description are very similar, but are not shown on the graph for clarity. At positive saturation, a maximal amount of energy is stored. This energy is released when going to the coercive state. Part of the energy is dissipated and part of the energy is sent back to the source. When going to negative saturation, exactly the same amount of energy is stored again in the system. This is clear from the cumulated stored energy density curve: it has a minimum at the coercive field value and is zero for positive as well as negative saturation. Since a part of the stored energy is sent to the source, the cumulated added energy density curve has negative values when going to the coercive state. However, from the moment $H_{a}=0.0 \mathrm{~A} / \mathrm{m}$, energy is flowing from the source towards the system, which results in a minimum at $H_{a}=0.0 \mathrm{~A} / \mathrm{m}$. The curve representing the cumulated dissipated energy density is strictly positive since the instantaneously dissipated energy is always positive. Both the cumulated added and stored energy density intersect at negative saturation, meaning that the net amount of energy which is added by 


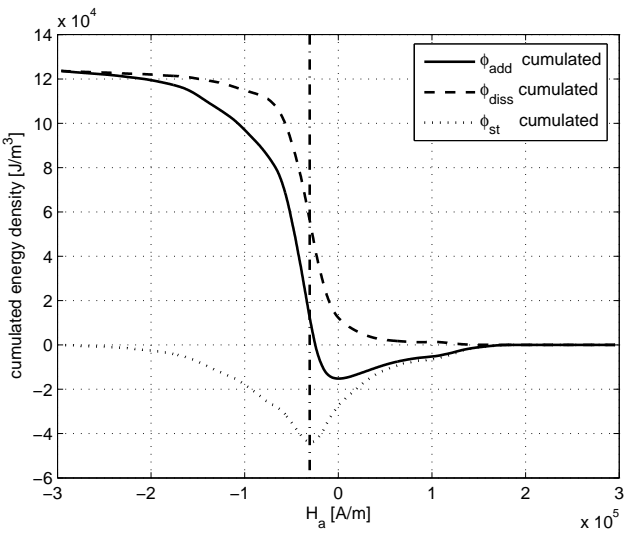

FIG. 14: Simulated hysteresis loop compared with the hysteresis loop reconstructed from the PDF. For completeness, the irreversible hysteresis loop is also shown.

the source when running through the hysteresis branch is all dissipated as heat by the ferromagnetic system.

The total dissipated energy density when running through the descending hysteresis branch equals $125.6 \mathrm{~kJ} / \mathrm{m}^{3}$ in the Preisach description and $128.8 \mathrm{~kJ} / \mathrm{m}^{3}$ in the micromagnetic description. As known, the total dissipated energy density when running through the complete hysteresis loop corresponds to the surface of the loop. Exploiting the symmetry in the hysteresis loop, it is clear that half the energy is dissipated when running through the descending branch and half of the energy is dissipated when running through the ascending branch. For the simulated hysteresis loop shown in Fig. 6 half of the surface equals $126.7 \mathrm{~kJ} / \mathrm{m}^{3}$. Hence, the values obtained in the micromagnetic and Preisach description differ only by about one percent from this number, showing the excellent agreement of the Preisach and the micromagnetic description on the one hand and the macro- scopic loss description based on the surface of the hysteresis loop on the other hand. In the Preisach model, this correspondence is incorporated theoretically in the model by construction. In the micromagnetic model, this result is something one expects based on simplified bistable systems as described in [15]. However, the excellent agreement for the complex multi-stable micromagnetic system considered here is striking.

\section{CONCLUSIONS}

A procedure is outlined to derive the Preisach distribution function from micromagnetic simulations. This is an interesting approach to study the relations between the microstructure of the material and the macroscopic magnetic hysteresis properties. A striking agreement is found between the micromagnetic and the Preisach description when comparing the (instantaneously) added, dissipated and stored energy density in the ferromagnetic system when running through the hysteresis loop. As a macroscopic property emanating from the micromagnetic description, the energy dissipated during one excitation cycle as computed in the micromagnetic model corresponds to the surface of the hysteresis loop. Moreover, the micromagnetic hysteresis model is able to provide a better insight in the instantaneous energy rearrangements. These results confirm that the micromagnetic hysteresis model is a valuable tool in the study of hysteresis properties and loss mechanisms in ferromagnetic materials.

\section{Acknowledgements}

This work was supported by FWO project G.0142.08 and GOA project BOF 07/GOA/006.
[1] F. Deng, "An improved iron loss estimation for permanent magnet brushless machines," IEEE Transactions on Energy Conversion, vol. 14, no. 4, pp. 1391-1395, 1999.

[2] J.W. Jung and K. Nam, " A vector control scheme for EV induction motors with a series iron loss model," IEEE Transactions on Industrial Electronics, vol. 45, no. 4, p. 617-624, 1998.

[3] D.C. Jiles, J.B. Thoelke, and M.K. Devine, "Determination of theoretical parameters for modelling bulk magnetic hysteresis properties using the theory of ferromagnetic hysteresis," IEEE Transactions on Magnetics, vol. 28, no. 27, 1992.

[4] H. Hauser, "Energetic model of ferromagnetic hysteresis," Journal of Applied Physics, vol. 75, no. 5, 1994.

[5] R.G. Harrison, "A physical model of spin ferromagnetism," IEEE Transactions on Magnetics, vol. 39, no. 2, pp. 950-960, 2003.

[6] F. Preisach, "Uber die magnetische Nachwirkung,"
Zeitschrift für Physik, vol.94, pp. 277-302, 1935.

[7] I. Mayergoyz, Mathematical models of hysteresis and their applications, New York: Springer, 2003.

[8] M.J. Sablik, "Modeling the effect of grain size and dislocation density on hysteretis magnetic properties in steels," Journal of Applied Physics, vol. 89, no. 10, pp. 5610-5613, 2001.

[9] G. Herzer, "Grain-size dependence of coercivity and permeability in nanocrystalline ferromagnets," IEEE Transactions on Magnetics, vol. 26, no. 5, pp. 1397-1402, 1990.

[10] G. Crevecoeur, P. Sergeant, L. Dupré, L. Vandenbossche, and R. Van de Walle, "Analysis of the local material degradation near cutting edges of electrical steel sheets," IEEE Transactions on Magnetics, vol. 44, no. 11, pp. 3173-3176, 2008.

[11] L.R. Dupré, G. Ban, M. Von Rauch, and J.A.A. Melkebeek, "Relation between the microstructural properties of electrical steels and the Preisach modelling," Journal 
of Magnetism and Magnetic Materials, vol. 195, no. 1, pp. 233-249, 1999.

[12] L. D. Landau and E. M. Lifshitz, Electrodynamics of continiuous media, Pergamom Press X: Oxford-London-New York-Paris, 1960.

[13] H. Kronmüller and M. Fänhle, Micromagnetism and the microstructure of ferromagnetic solids, Cambridge University Press: Max-Planck-Institut für Metallfoschung, Stuttgart, Germany, 1994.

[14] B. Van de Wiele, F. Olyslager, and L. Dupré, "Fast numerical three-dimensional scheme for the simulation of hysteresis in ferromagnetic grains," Journal of Applied Physics, vol. 101, 073909, 2007.

[15] G. Bertotti, Hysteresis in Magnetism, Academic Press, 1994.

[16] O. Bottauscio, and A. Manzin, "Critical aspects in micromagnetic computation of hysteresis loops of nanometer particles," IEEE Transactions on Magnetics, vol. 45, no. 11, 2009.

[17] W.F. Brown Jr., "Thermal fluctuations of a singledomain particle," Physical Review, vol. 130, no. 5, pp. 1677-1686, 1963.

[18] P.S. Krishnaprasad and X. Tan, "Cayley transforms in micromagnetics," Physica B, vol. 306, pp. 195-199, 2001

[19] P.B. Monk and O. Vacus, "Accurate discretization of a nonlinear micromagnetic problem," Computational Methods in Applied Mechanics and Engineering, vol. 190, no. 40-41, pp. 5243-5269, 2001

[20] C. Serpico, I.D. Mayergoyz, and G. Bertotti, "Numerical technique for integration of the Landau-Lifshitz equation," Journal of Applied Physics, vol. 89, pp. 6991-6993, 2001.

[21] A.W. Spargo, P.H.W. Ridley, and G.W. Roberts, "Geometric integration of the Gilbert equation," Journal of Applied Physics, vol. 93, pp. 6805-6807, 2003.

[22] M. d'Aquino, C. Serpico, and G. Miano, G., "Geometrical integration of Landau-Lifshitz-Gilbert equation based on the mid-point rule," Journal of Computational Physics, vol. 209, pp. 730-753, 2005.
[23] B. Van de Wiele, F. Olyslager, and L. Dupré, "Fast semianalytical time integration schemes for the LandauLifshitz equation," IEEE Transactions on Magnetics, vol. 43, no. 6, pp. 2017-2019, 2007.

[24] G. Brown, T.C. Schulthess, D.M. Apalkov, and P.B. Visscher, "Flexible fast multipole method for magnetic simulations," IEEE Transactions on Magnetics, vol. 40, no. 4, pp. 2146-2149, 2004.

[25] A. Vansteenkiste, M. Weigand, M. Curcic, H. Stoll, G. Schütz, and B. Van Waeyenberge, "Chiral symmetry breaking of magnetic vortices by sample rougness," New Journal of Physics, vol. 11, art.no. 063006, 2009.

[26] B. Van de Wiele, F. Olyslager, and L. Dupré, "Application of the fast multipole method for the evaluation of magnetostatic fields in micromagnetic computations," Journal of Computational Physics, vol. 227, pp. 99139932, 2008

[27] E.E. Ong, K.M. Lim, and H.P. Lee, "A fast algorithm for three-dimensional potential fields calculation: fast Fourier transform on multipoles," Journal of Computational Physics, vol. 192, pp. 244-261, 2003.

[28] V. Basso, G. Bertotti, A. Infortuna, and M. Pasquale, "Preisach model study of the connection between magnetic and microstructural properties of soft magnetic elements," IEEE Transactions on Magnetics, vol. 31, no. 6, pp. 4000-4005, 1995.

[29] L. Vandenbossche, M.J. Konstantinović, and L. Dupré, "Magnetic hysteretic characterization of the irradiationinduced embrittlement of $\mathrm{Fe}$, Fe- $\mathrm{Cu}$ model alloys, and reactor pressure vessel steel," Journal of Magnetism and Magnetic Materials, Vol. 320, pp. e562-e566, 2008.

[30] P.A. Voltairas, D.I. Fotiadis, and C.V. Massallas, "Magnetization reversal in thin ferromagnetic films under mechanical stress," International Journal of Engineering Science, vol. 38, pp. 903-919, 2000.

[31] D. Everett, "A general approach to hysteresis - part4, an alternative formulation of the domain model," Transactions Faraday Society, vol. 51, pp. 1551-1557, 1955. 